\title{
Acetamidinium substituted methylammonium lead iodide perovskite solar cells with higher open-circuit voltage and improved intrinsic stability
}

\author{
Pallavi Singh†, Rudra Mukherjee†, Sushobhan Avasthi*
}

Center for Nanoscience and Engineering, Indian Institute of Science, Bengaluru, India560012

$\dagger$ Both authors contributed equally

*Email id: savasthi@iisc.ac.in

\section{Contents}

1. Figure S1. Indexing, refinement and whole pattern fitting of $\mathrm{AAPbI}_{3} \mathrm{XRD}$ pattern using JADE software

2. Table S1. Change in lattice parameter and bandgap of perovskite thin film with varying AA composition.

3. Figure S2. (a) Tauc plot of films with $0-20 \%$ AA composition (b-d) magnified tauc plots of $0 \%, 10 \%$ and $20 \%$ AA substituted films.

4. Figure S3. (a) UPS spectra of as-deposited $0-20 \%$ AA substituted films (b-g) work function and difference between fermi level and valence band maximum of $0-20 \%$ AA substituted films.

5. Table S2. Position of Valence band maximum and Conduction band minimum from UPS measurement.

6. Figure S4. Line Diagram of Microwave Resonance-assisted Photoconductivity measurement setup. $\mathrm{s}$

7. Figure S5. JV of $20 \%$ AA substituted solar cell

8. Figure S6. Top-surface FESEM images of perovskite thin film with varying AA composition. 


\section{Indexing, refinement and whole pattern fitting of $\mathrm{AAPbI}_{3} \mathrm{XRD}$ pattern}

Scan ID: AAPbI3-as-dep_Theta_2-Theta.raw

Scan Parameters: $\quad 5.0 \% 60.0 \% 01 \%(\mathrm{~s}), \mathrm{I}(\mathrm{p})=13917 / 166, \mathrm{Cu}(40 \mathrm{kV}, 30 \mathrm{~mA})$, Saturday, January $11,2020,2: 31 \mathrm{PM}$

\begin{tabular}{|c|c|c|c|}
\hline$\checkmark$ Zero Offset $=-0.0005(0.0015)$ & Displacement $=0.0$ & & Beam Spill-Off below $0.0^{\circ}$ \\
\hline$\checkmark$ Ka2 Peaks Present & Ka2/Ka1 Ratio $=0.5$ & & X-Ray Polarization $=1.0$ \\
\hline Geometry: Diffractometer Lp & Fitted-Range: $\quad 10.0^{\circ}-50.0^{\circ}$ & BG-Model: & $\lambda: \quad 1.54059 \AA(\mathrm{Cu})$ \\
\hline
\end{tabular}

PSF: pseudo-Voigt Broadening: Individual FWHM Curve Instrument: Constant FWHM $=0.1^{\circ}$

\begin{tabular}{|l|c|c|c|c|c|c|c|c|}
\hline Phase ID (1) & Chemical Formula & PDF-\# & Wt\% (ESD) & RIR & $\mu$ & a (ESD) & c (ESD) & $a(E S D)$ \\
\hline Hexagonal • R-3r & Pattern Indexing & $100.0(0.8)$ & $(1.0)$ & 0.0 & $15.62196(155)$ & $10.04132(115)$ & $90.00(0)^{\circ}$
\end{tabular}

Refinement Converged $(R / E=1.2)$, Round $=4$, Iter=3, $P=50, R=5.32 \%(E=4.42 \%, E P S=0.5)$

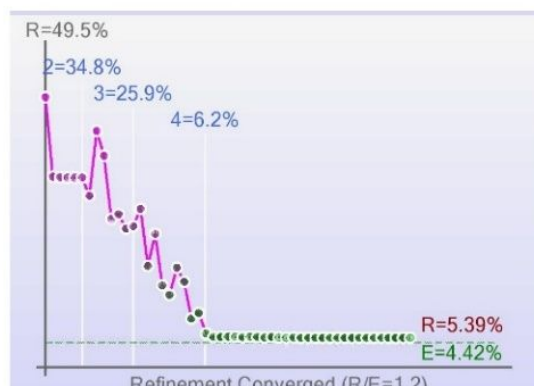

\Hexagonal $\bullet$ R-3m (166):...

Refinement Converged (R/E=1.2)
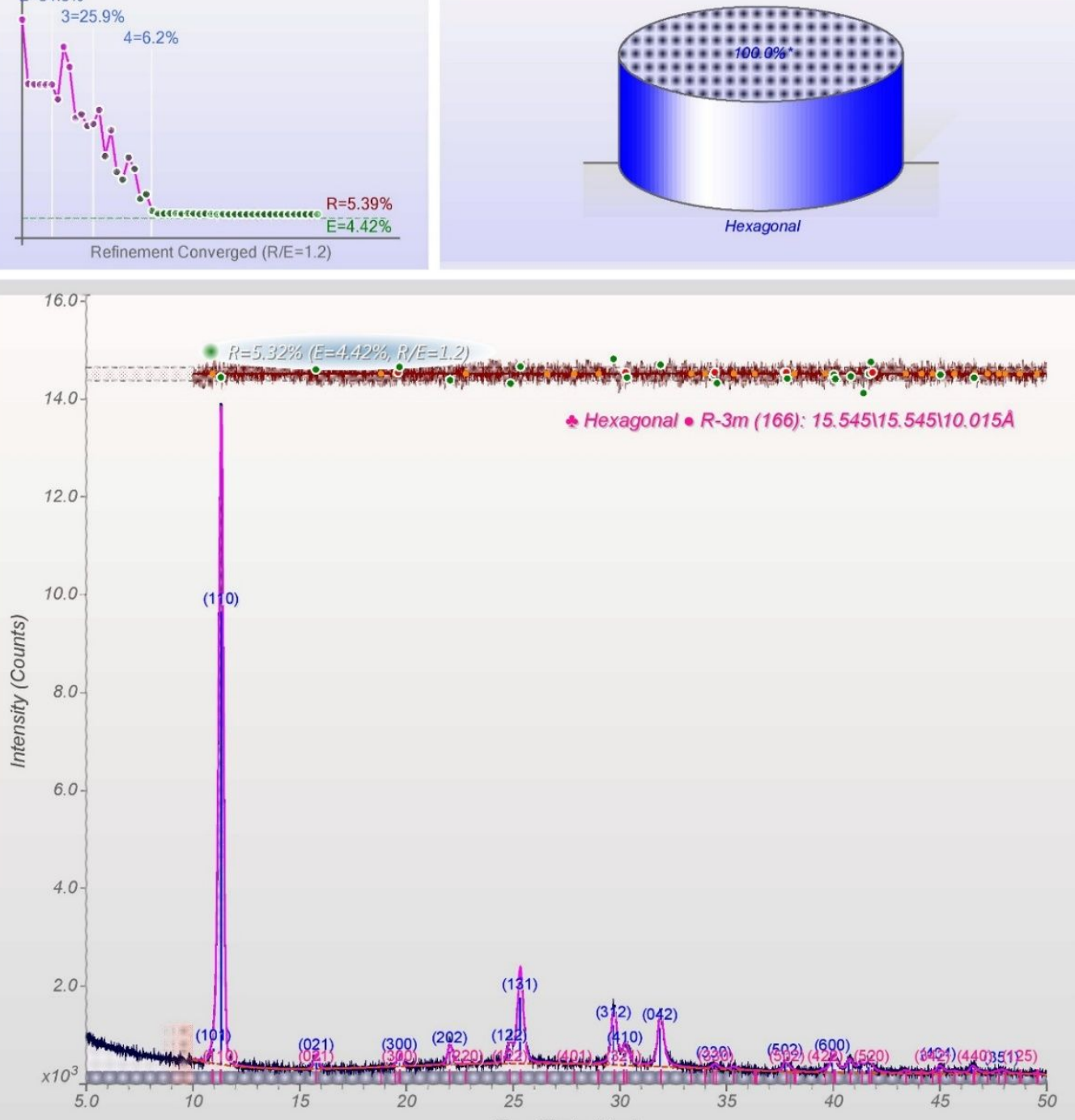

Figure S1. Indexing, refinement and whole pattern fitting of $\mathrm{AAPbI}_{3} \mathrm{XRD}$ pattern using JADE software 
The pattern indexing, refinement and whole pattern fitting (WPF) of diffraction pattern of $\mathrm{AAPbI}_{3}$ is done by Jade software using an MDI proprietary algorithm. The outcome is a hexagonal unit cell (space group: R-3m (166)) with lattice parameters a and c $15.545 \AA$ and $10.041 \AA$ respectively.

JADE performs least-squares refinements on the possible cells from the line positions (fitting range: 10-50 $2 \theta$ value) based on figure-of-merit (range : 5 to 95) to choose the most likely space group followed by whole pattern fitting on the chosen space to produce comparatively precise lattice parameters than those from the least-square fit. The $\mathrm{R}$ (weighted)/E(experimental) ratio obtained is 1.2 (for ideal refinement $\mathrm{R} / \mathrm{E}=1$ ) ensuring a good fit.

Table S1. Change in lattice parameter and bandgap of perovskite thin film with varying AA composition.

\begin{tabular}{lllll}
\hline Composition & $\begin{array}{l}\text { Lattice Parameter } \\
\mathrm{a}[\mathrm{A}]\end{array}$ & $\begin{array}{l}\text { Lattice } \\
\mathrm{c}[\mathrm{A}]\end{array}$ & Parameter & $\begin{array}{l}\text { Volume } \\
{\left[\mathrm{A}^{3}\right]}\end{array}$ \\
\hline $0 \% \mathrm{AA}$ & 8.890 & 12.73 & 1006.25 & 1.57 \\
$10 \% \mathrm{AA}$ & 8.923 & 12.78 & 1017.70 & 1.58 \\
$20 \% \mathrm{AA}$ & 8.928 & 12.79 & 1019.49 & 1.58 \\
$\mathrm{AAPbl}$ & - & - & - & 2.32 \\
\hline
\end{tabular}



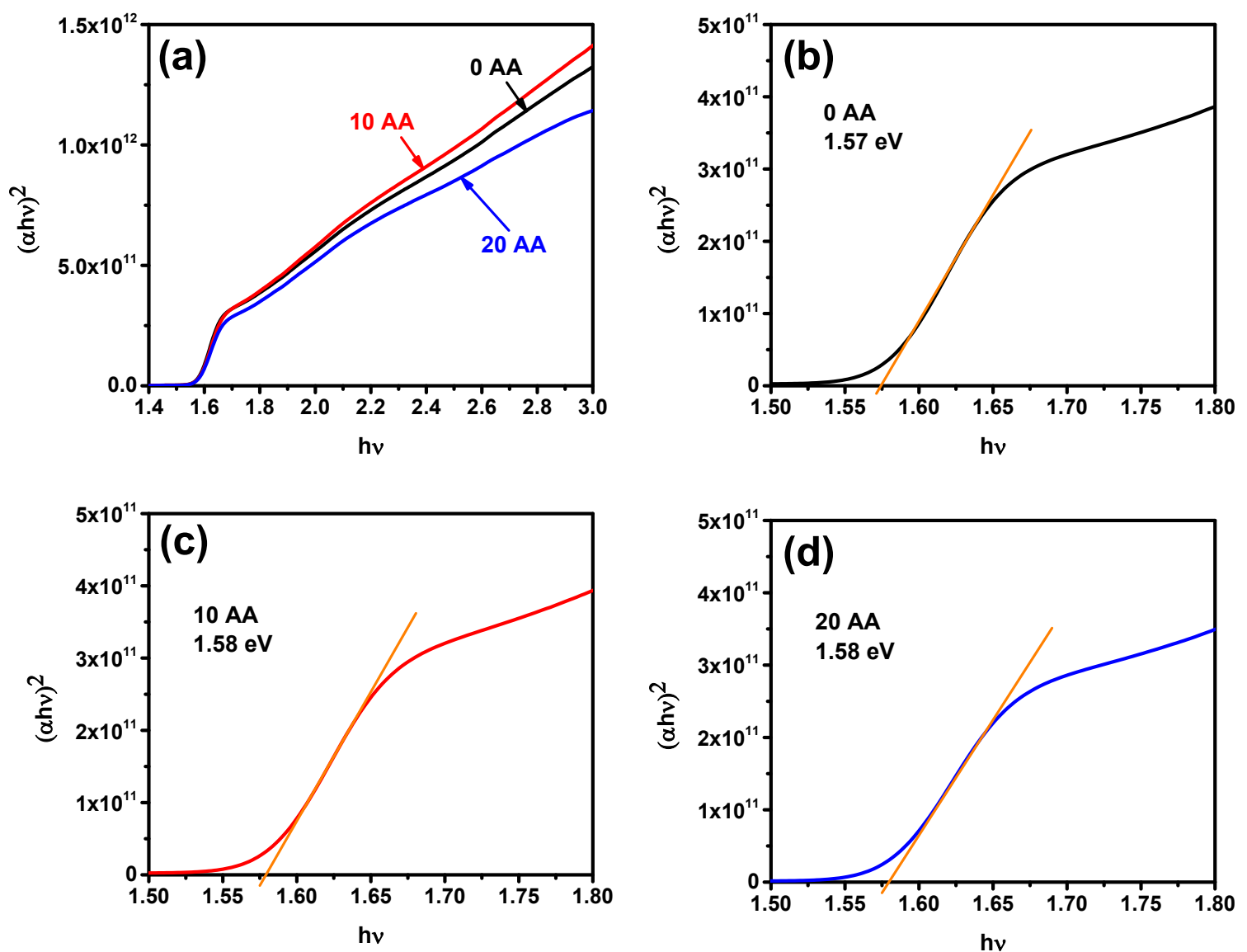

Figure S2. (a) Tauc plot of films with $0-20 \%$ AA composition (b-d) magnified tauc plots of $0 \%, 10 \%$ and $20 \%$ AA substituted films. 

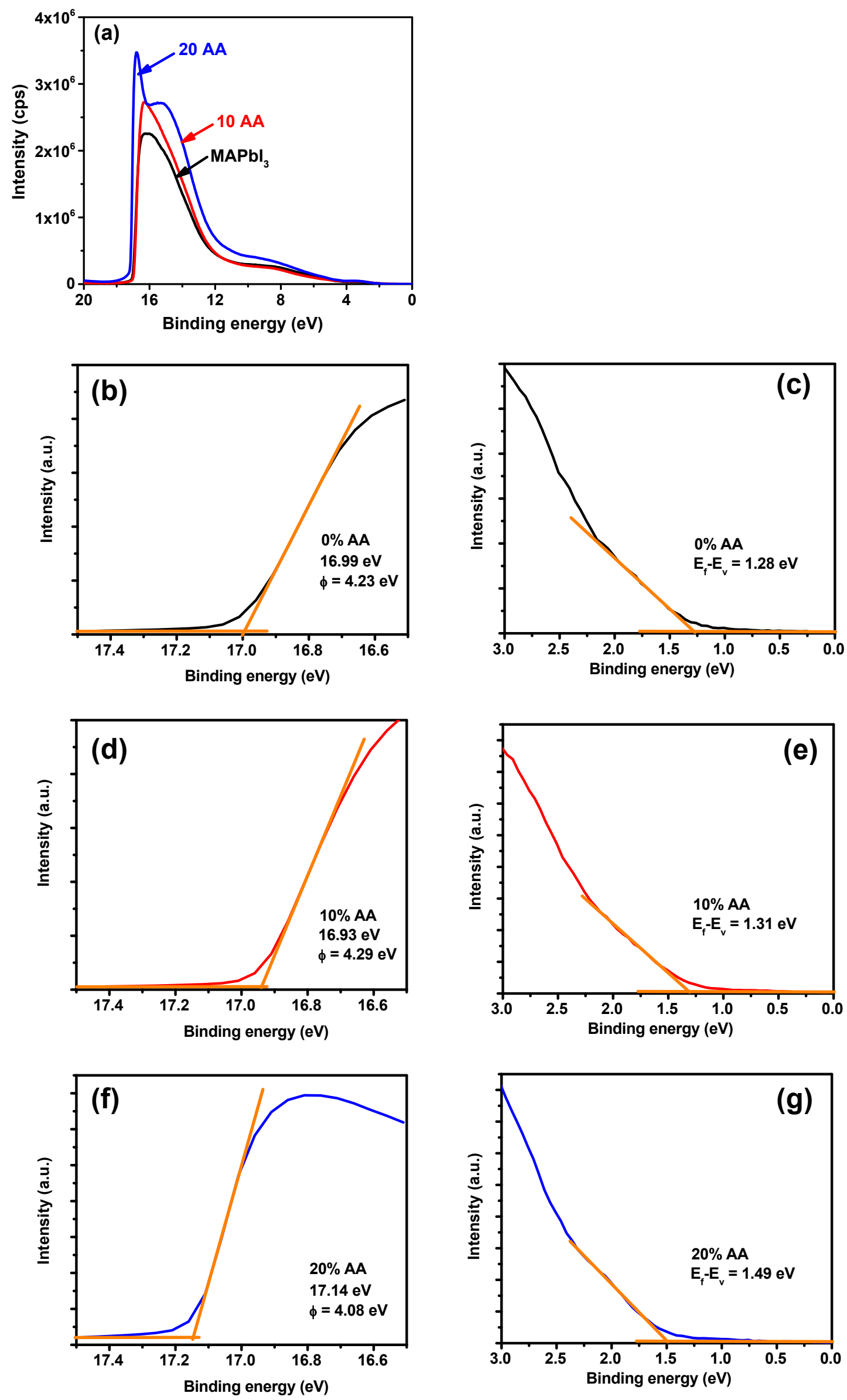

Figure S3. (a) UPS spectra of as-deposited 0-20\% AA substituted films (b-g) work function and difference between fermi level and valence band maximum of 0-20\% AA substituted films. 
Table S2. Position of Valence band maximum and Conduction band minimum from UPS measurement.

\begin{tabular}{lllll}
\hline Composition & $\begin{array}{l}\text { Work Function } \\
(\Phi)\end{array}$ & $\mathrm{E}_{\mathrm{F}-\mathrm{E}_{\mathrm{V}}}$ & $\mathrm{VBM}$ & $\mathrm{CBM}$ \\
\hline $0 \% \mathrm{AA}$ & 4.23 & 1.28 & -5.51 & -3.94 \\
$10 \% \mathrm{AA}$ & 4.29 & 1.31 & -5.6 & -4.02 \\
$20 \% \mathrm{AA}$ & 4.08 & 1.49 & -5.57 & -3.99 \\
& & & & \\
\hline
\end{tabular}

\section{Microwave Detected Photoconductivity}

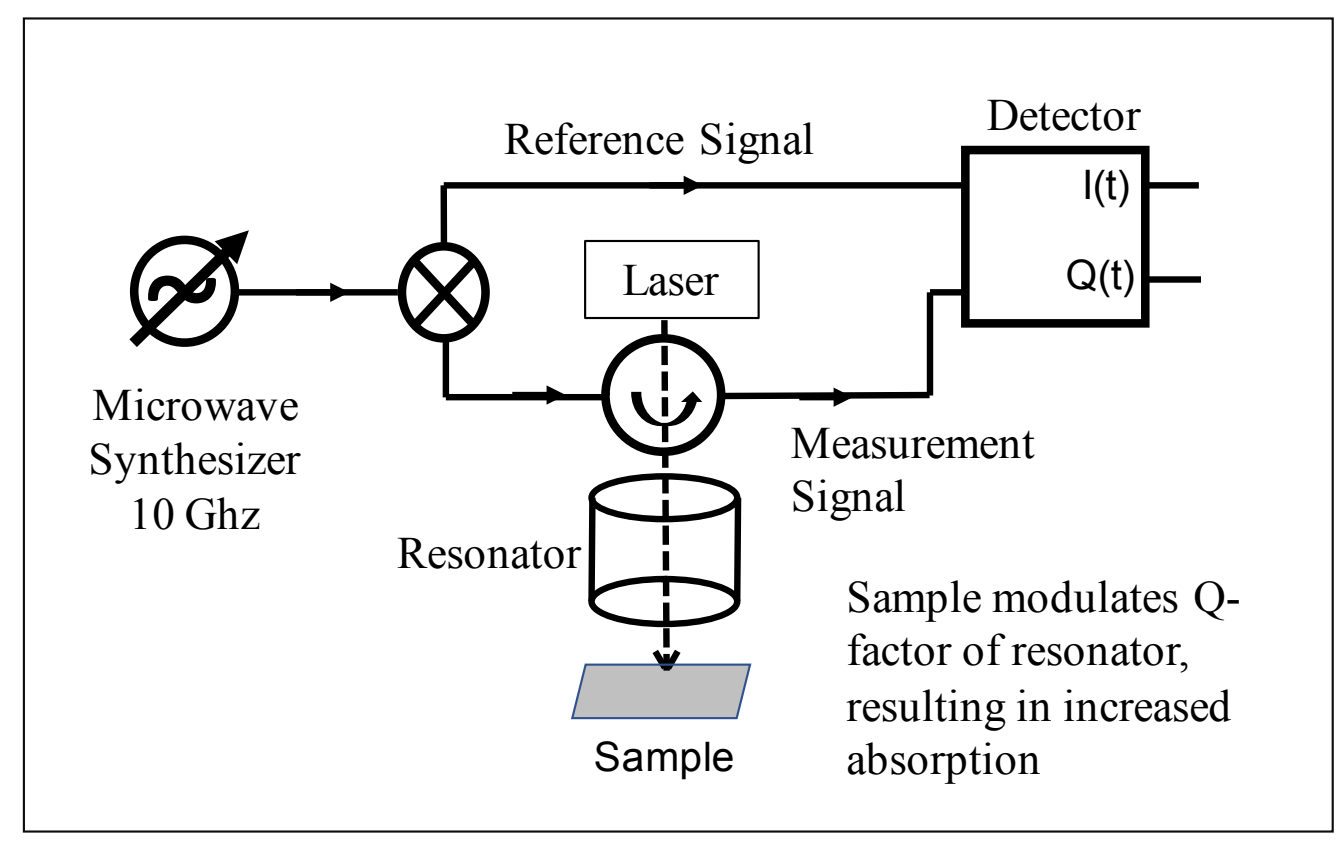

Figure S4. Line Diagram of Microwave Resonance-assisted Photoconductivity measurement setup.

The tool uses a microwave resonance chamber to enhance the signal. The quality factor of the resonance chamber depends on the losses in the chamber walls and the sample. The sensitivity of the setup added with the relaxation in the time scale till which the transient state can be captured and low excitation ranges allows the measurement of carrier lifetimes in $\sim 1-10$ sun 
illumination as compared to $\sim 10^{4}$ sun illumination in conventional TRPL setup. At high excitation ranges (as in TRPL) the auger and radiative recombination mechanisms dominate while in an actual solar cell (under 1 sun) the non-radiative (SRH) recombination will have a higher share and hence MDP is a better emulation of the actual solar cell environment as compared to the conventional techniques such as $\mu$ PCD and TRPL.

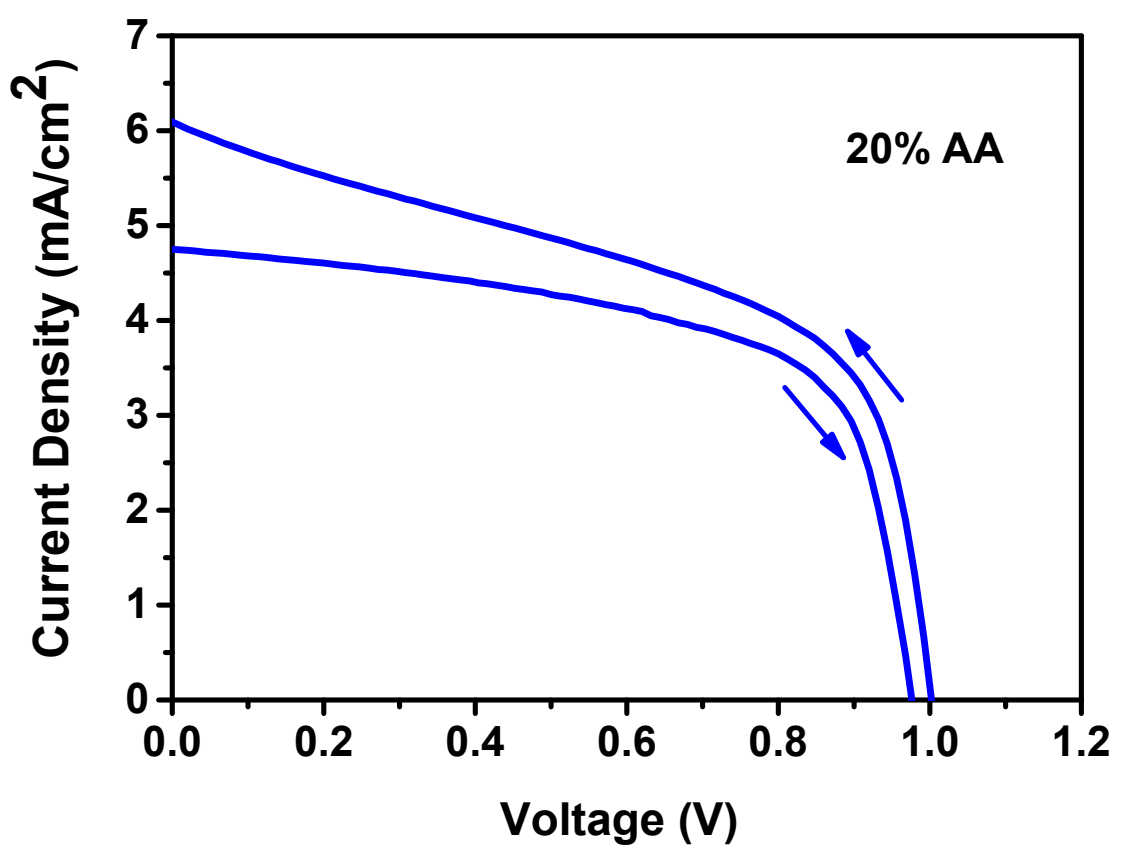

Figure S5. JV of 20\% AA substituted solar cell
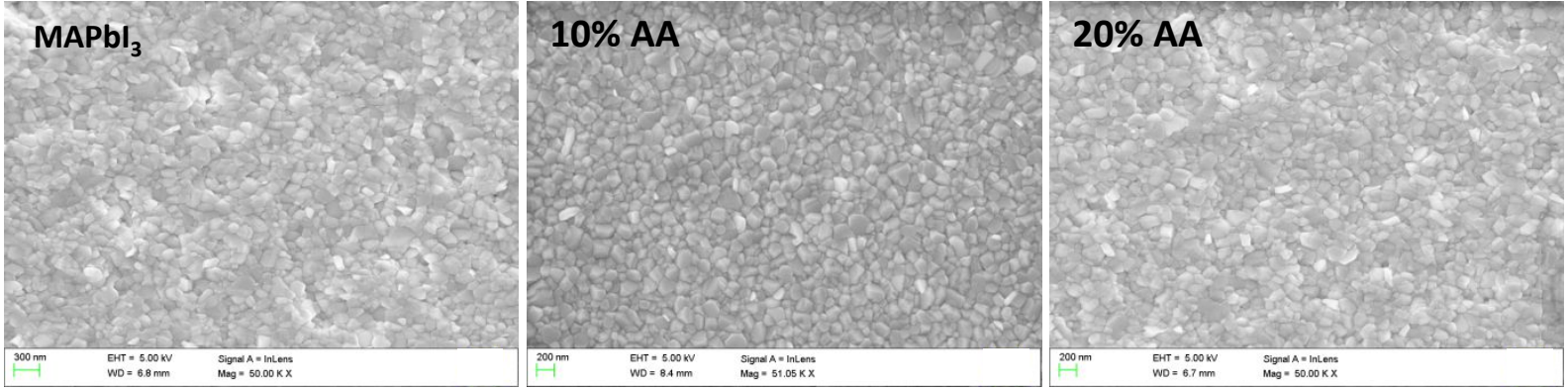

Figure S6. Top-surface FESEM images of perovskite thin film with varying AA composition. 